\title{
EXAMINATION OF THE LBM IN SIMULATION OF MICROCHANNEL FLOW IN TRANSITIONAL REGIME
}

\author{
Ching Shen., Dong-Bo Tian, Chong Xie, and Jing Fan \\ Institute of Mechanics, Chinese Academy of Science \\ Zhonguancun Road, Beijing, China, 100080, cshen@imech.ac.cn
}

\begin{abstract}
Gas flows in micro-electro-mechanical systems (MEMS) owing to the small size of the systems possess a relatively large Knuden number and usually belong to the slip and transitional flow regimes. This paper employs three schemes, namely the direct simulation Monte Carlo (DSMC) method, information preservation (IP) method, and the lattice Boltzmann method (LBM), to simulation micro-channel flows at three Knudsen numbers $(K n)$ of $0.0194,0.194$ and 0.388 . The present LBM results are in agreement with those given by Nie et al. (2002), whereas they significantly differ from the DSMC (and IP) results as $K n$ increases. This suggests that the present version of LBM is not feasible to simulate the micro-channel flows in transition regime.
\end{abstract}

\section{INTRODUCTION}

The end of $20^{\text {th }}$ century marks the beginning of the manufactory of micro-electro-mechanical systems (MEMS) and the study of the flows in them (Ho \& Tai 1998). The space and time scales of MEMS are at order of micron and microsecond, respectively. There are various MEMS devices manufactured by micromachining technique, such as micro channels, micro pumps, micro nozzles, micro valves, micro-motors etc. The gas flows in MEMS owing to the small size of the systems possess a relatively large Knudsen number $K n=\lambda / \mathrm{L}$, and usually fall into the slip transitional flow regimes, where $\lambda$ is the molecular mean free path and $\mathrm{L}$ is the characteristic length of flow. Application of the methods of molecular gas dynamics or rarefied gas dynamics is inevitable. Except in the micro spacecraft applications the gas flows in MEMS are typically of low speed, say of order of $10 \mathrm{~cm} / \mathrm{s}$ (cf. Pong et al. 1994; Ho
\& Tai 1998). Another feature of micro channel flows is the large aspect ratio (the ratio of the channel length to height). For example, in micro-channel experiments measuring the stream-wise pressure distribution carried out by Pong et al. (1994) and Shi et al. (1996), the dimensions are $1.2 \mu \mathrm{m} \times 30 \mu \mathrm{m} \times 3000 \mu \mathrm{m}$ and $1.2 \mu \mathrm{m}$ $\times 40 \mu \mathrm{m} \times 4000 \mu \mathrm{m}$, respectively. The low flow velocity causes the large ratio of noise to useful information, which requires a very large sampling size in conventional statistical simulation approaches such as the direct simulation Monte Carlo (DSMC) method (Bird 1994). And also, because of the low velocity the boundary value problem of the channel flow is of the elliptic nature, and the velocity and pressure at the inlet and exit that are dependent each other must be regulated until the calculation converges to a steady state. The large aspect ratio makes this regulation very difficult for the DSMC calculations. An information preservation (IP) method was proposed to more efficiently calculate MEMS flows than DSMC (Fan \& Shen 1999, 2001). This method has been successfully applied to many micro flows (Fan \& Shen 1999, 2001; Fan, et al. 2001; Cai, et al. 2000; Sun, Boyd, Candler 2002; Sun \& Boyd 2002; Jiang, Fan \& Shen 2002; Xie, Fan \& Shen 2002). Recently, the lattice Boltzmann method (LBM) was also attempted to simulate gas flows in micro-channel and cavity (Nie et al. 2002), and in high Knudsen number channel flows negative pressure deviations from a linear pressure drop was observed.

In this paper, firstly, various tools in simulation of MEMS flows are reviewed. Then the version of LBM for MEMS (Nie et al., 2002) that is employed here is briefly described. Next, micro-channel gas flows in the slip and transitional regimes are calculated using DSMC, IP and LBM, with an emphasis on examination of the negative pressure deviations from a linear pressure drop in 
transition regime observed in the LBM calculation of Nie et al. (2002). Finally some conclusions are given.

\section{TOOLS IN SIMULATING MEMS GAS FLOWS}

In the arsenal for simulating slow rarefied gas flows there are the Navier-Stokes equations with slip boundary conditions, the linearized Boltzmann equation, the DSMC method, the IP method and LBM.

By its nature the Navier-Stokes equation with slip boundary conditions is appropriate for small Knudsen number $(K n<0.1)$. Recently Kardiadakis and Beskok (2001) proposed high order slip model and obtained density and pressure distributions, velocity profiles and slip velocity for plane Poiseuille flow in agreement with the results of DSMC and linearized Boltzmann equation for $K n$ equal up to 0.2 . The unified flow model developed by them based on velocity scaling law for transitional flow is a kind of formula expression of the DSMC and linearized Boltzmann results but could not serve as a general method in the transitional regime.

The linearized Boltzmann equation method (Cercignani 1998; Ohwada, Sone \& Aoki 1989) is suitable for solving the low speed rarefied gas flows and its solutions for benchmark problems may serve as criteria of testing other methods. But the complexity in performing the numerical solution of the Boltzmann equation limits most existing solutions to simple geometry. Sometimes the equation being linearized is not the Boltzmann equation but the BGK equation (Bhatnangar, Gross \& Krook 1954). The BGK solution is relatively easier, but some correction must be made to match physical reality. Generally, there are small discrepancies between the BGK and real molecule model solutions (Sharipov \& Seleznev 1998; Jiang, Shen and Fan, this conference).

The DSMC method (Bird, 1994) is an appropriate means to treat rarefied gas flow, and has been verified by the comparisons both of global characteristics and of velocity distribution level characteristics of high speed flow fields with experimental results. The converged (with sufficient sample size) DSMC simulation can be used to test the validity of other methods. But the statistical fluctuations inherent of the DSMC method make the useful information drowned by the huge background noise when simulating low speed gas flows in MEMS. The requirement for the time step to be less than the collision time makes the task of regulating the boundary conditions at the inlet and the outlet of the long channels unaccomplishable. So nowadays only simulations of high speed channel flows or short channel flows can be seen in the literature. This method is used to test the validity of the LBM calculation, as the example considered here is a case of not so large an aspect ratio (100:1) and DSMC can yield convergent results with sufficient sample size.

The IP method (Fan and Shen, 1999, 2001) is a method embedded in DSMC method and uses the simulated molecules to carry the information of an enormous number of molecules that a simulated molecule represents, the information characteristics vary according to the conservation laws and are summed to give macroscopic quantities. The convergence time is shortened immensely. In the simulation of bench mark unidirectional (Couette, Poiseuille, and Rayleigh) flows IP method has been verified by the results of linearized Boltzmann equation method and the DSMC simulation and can capture the Knudsen minimum in Poiseuille flows. In simulating the long channel flows where experimental investigations (Pong et al. 1994; Shi et al. 1996; Arkilic, Schmidt \& Breuer 1997) have been made, the IP simulation (Xie, Fan \& Shen 2002; Shen,Fan \& Xie,2003) yields the pressure distributions and mass flow rates in good agreement with the experimental data. The issue of regulation of the boundary conditions (the inlet and outlet pressure and velocity) at the entrance and exit of the long channel was successfully settled by using the conservation form of the mass continuity equation and the super relaxation method. This has general significance for MEMS flows. A general implementation of the method including 2-D and 3-D cases was elucidated in a recent monograph (Shen, 2003) and in (Shen, Fan \& Xie,2003). In this paper the IP method is also utilized to simulate the cases of interest for comparison.

LBM (Chen and Doolen 1998) solves the simplified Boltzmann equation on regular lattice points. The LBM solution converges to the Navier-Stokes solution for small $\mathrm{Kn}$. There has been an attempt to use it in the transitional regime (Nie, Doolen \& Chen 2002). It is of great interest to use LBM to simulate gas flows in MEMS for its ease in handling complex geometry, simplicity in implementation and its high efficiency. The validity of this has not been verified. The purpose of the present paper is to test the feasibility of using the LBM to simulate transitional flow.

\section{THE RECENT VERSION OF LBM FOR SIMULATION OF FLUID FLOW IN MEMS}

In the recent version of the LBM (Nie, Doolen, and Chen, 2002) the relaxation parameter $\tau$ in the Lattice Boltzmann equation with BGK collision approximation is modified as

$$
\tau^{\prime}=\frac{1}{2}+\frac{1}{\rho}\left(\tau-\frac{1}{2}\right),
$$

and the kinematic viscosity $v$ is expressed as

$$
v=\mathrm{c}_{\mathrm{s}}^{2}(2 \tau-1) / 2 \rho,
$$


where $\rho$ is the density and $c_{s}$ is the sound speed of the gas. The mean free path is defined as

$$
\lambda=\mathrm{a}(\tau-0.5) / \rho
$$

where a is constant and is chosen to be 0.388 to match the simulated results and experimental data of mass flow rates. Therefore, for a characteristic length of $h$, the Knudsen number may be written as

$$
K n=\frac{\mathrm{a}(\tau-0.5)}{\rho \mathrm{h}} .
$$

According to the D2Q9 lattice model (Qian, d'Humi éres \& Lallemand 1992), the lattice BGK equation can be expressed as

$$
\mathrm{f}_{\mathrm{i}}\left(\mathbf{r}+\mathbf{c}_{\mathrm{i}} \delta \mathrm{t}, \mathrm{t}+\delta \mathrm{t}\right)-\mathrm{f}_{\mathrm{i}}(\mathbf{r}, \mathrm{t})=-\frac{\mathrm{f}_{\mathrm{i}}-\mathrm{f}_{\mathrm{i}}^{\mathrm{eq}}}{\tau},
$$

with the equilibrium distribution

$$
\mathrm{f}_{\mathrm{i}}^{\mathrm{eq}}=\mathrm{t}_{\mathrm{i}} \rho\left[1+\frac{\mathrm{c}_{\mathrm{i} \alpha} \mathrm{u}_{\alpha}}{\mathrm{c}_{\mathrm{s}}^{2}}+\frac{\left(\mathrm{c}_{\mathrm{i} \alpha} \mathrm{c}_{\mathrm{i} \beta}-\mathrm{c}_{\mathrm{s}}^{2} \delta_{\alpha \beta}\right)}{2 \mathrm{c}_{\mathrm{s}}^{4}} \mathrm{u}_{\alpha} \mathrm{u}_{\beta}\right] \text {, }
$$

where $\mathrm{c}_{\mathrm{s}}=1 / \sqrt{3}, \mathrm{t}_{0}=4 / 9, \mathrm{t}_{\mathrm{i}}=1 / 9$ for $\mathrm{i}=1,2,3,4$, $\mathrm{t}_{\mathrm{i}}=1 / 36$ for $\mathrm{i}=5,6,7,8, \alpha$ and $\beta$ denote the Cartesian directions, and the fluid density $\rho$ and velocity $\mathbf{u}$ are defined as

$$
\rho=\sum_{i} f_{i}, \quad \rho \mathbf{u}=\sum_{i} \mathbf{c}_{i} f_{i} .
$$

The bounce-back boundary condition is employed along a solid wall: when a particle reaches a lattice node in a layer adjacent to and inside the wall it scatters back to the fluid nodes along its incoming direction. The densities at the channel inlet and exit are known as $\rho_{i}$ and $\rho_{e}$, respectively, while an extrapolation scheme (Chen, Martinez \& Mei 1996) is used to regulate the pressure distribution among the lattice nodes.

\section{RESULTS AND DISCUSSION}

Consider gas flows through a two-dimensional micro channel. To be consistent with the computational conditions of Nie et al. (2002), three cases are studied, with the Knudsen number of 0.0194, 0.194, and 0.388, respectively, while the ratio of the channel length to height $L / h$ keeps 100 . The inlet and outlet pressure ratio $\mathrm{p}_{\mathrm{i}} / \mathrm{p}_{\mathrm{e}}$ is 2 and 1.4 , respectively. Three numerical schemes including DSMC, IP, and LBM are employed.
The details of the DSMC and IP implementation for micro-channel flows are described in (Xie, Fan \& Shen 2002), Shen(2003) and (Shen, Fan \& Xie, 2003).

Figure 1 shows the mass flow rates as a function of $K n$ given by three methods. They generally agree well each other, though the LBM value is slightly higher at $K n=0.388$. This is understandable, because in the version of LBM for MEMS flows the constant $a$ in Eq. (3) is adjusted to best match measured data of mass flow rate (Nie et al., 2002).

Figures 2-7 show the normalized velocity profiles and the deviation of the stream-wise pressure from a linear pressure distribution at different Knudsen numbers, where $U_{\max }$ is the maximum velocity, $\mathrm{p}_{1}=\mathrm{p}_{\mathrm{e}}+\left(\mathrm{p}_{\mathrm{i}}\right.$ $\left.-\mathrm{p}_{\mathrm{e}}\right)(1-\mathrm{X})$, and $X=x / L$. In the slip regime with $K n=0.0194$, the velocity profiles at the exit given by LBM, DSMC, IP and slip Navier-Stokes equations agree well with each other (Fig. 2), and the four pressure distributions are unanimous in trend but slightly different in details (Fig.3). The sample sizes of DSMC and IP calculations are given in Table 1. It takes about 5 days on a Pentium-IV $1.5 \mathrm{G}$ PC so as to obtain an enormous sample size of DSMC. At this sample size, the DSMC trend can be seen whereas the statistical scatter is still obvious.

For $K n=0.194$, the velocity profiles at the exit obtained by LBM shows minor difference from those obtained by the other three methods (Fig.4). A more apparent difference appears in the pressure distribution

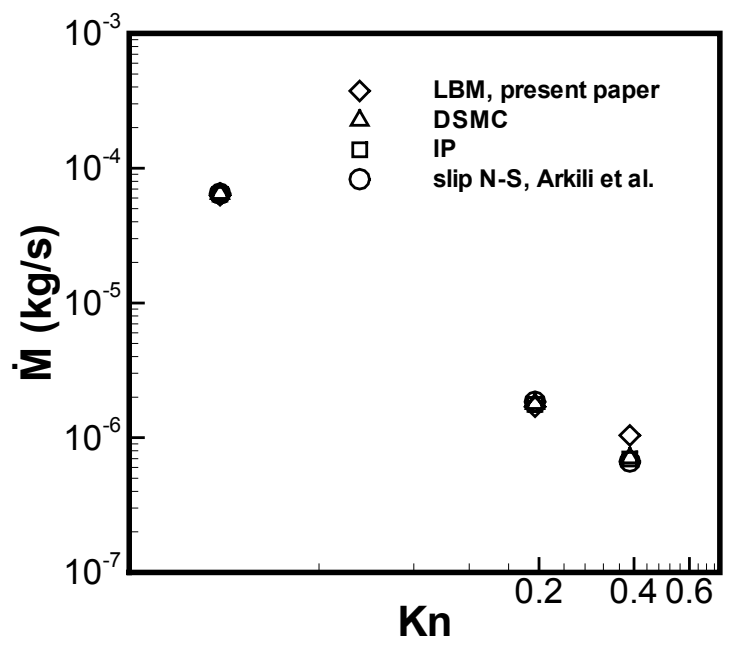

Fig. 1 The mass flow rate versus the Knudsen number at the exit. 
(Fig.5): the LBM result swings closely around the null line, while the DSMC and IP results show clear large positive deviation from the linearity.

For $K n=0.388$ in the transitional regime, the LBE velocity profile significantly deviates from the DSMC and IP profiles (Fig.6), and the negative behavior of $\left(\mathrm{p}-\mathrm{p}_{1}\right) / \mathrm{p}_{\mathrm{e}}$ predicted by LBM essentially differ from the positive prediction of DSMC and IP (Fig.7). This clearly shows the unfeasibility of LBM in simulating MEMS flow in transitional flow

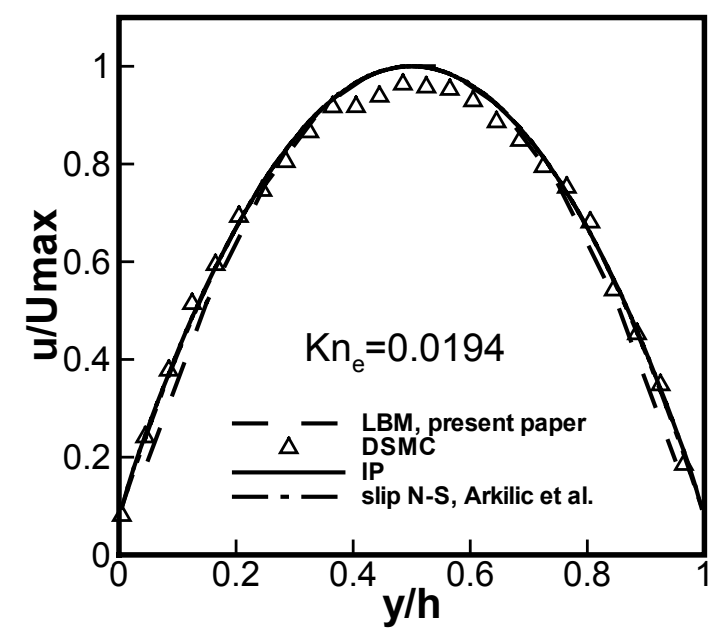

Fig. 2 Comparison of the LBM, DSMC, IP, and slip Navier-Stokes velocity profiles at the channel exit for $K n=0.0194$ and $\mathrm{p}_{\mathrm{i}} / \mathrm{p}_{\mathrm{e}}=1.4$.

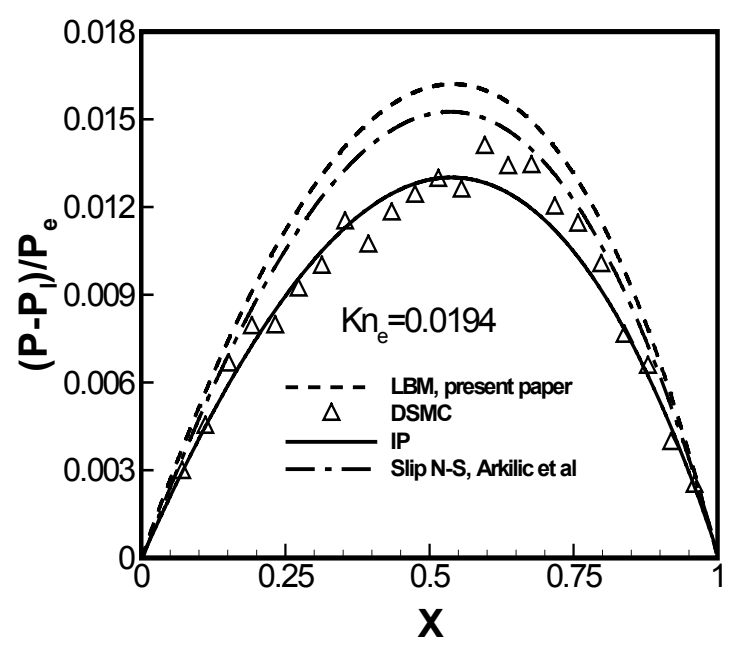

Fig. 3 Comparison of the deviation of the stream-wise pressure from a linear pressure distribution given by LBM, DSMC, IP, and slip Navier-Stokes equation.

\section{CONCLUSION}

It seems very tempting to use the Lattice Boltzmann method (LBM) to simulate gas flows in MEMS. On an example of microchannel of length to height ratio of 100 the present study reveals that LBM is suitable to simulate MEMS flows only for very small Knudsen numbers, here it retains certain advantages over conventional method as the handling complex geometry, simplicity in implementation and high efficiency in this near continuum regime. For Knudsen number as large as $K n=0.2$, LBM results deviate from DSMC results apparently. In the transitional flow regime, for $K n=0.388$ LBM results deviate from DSMC results significantly

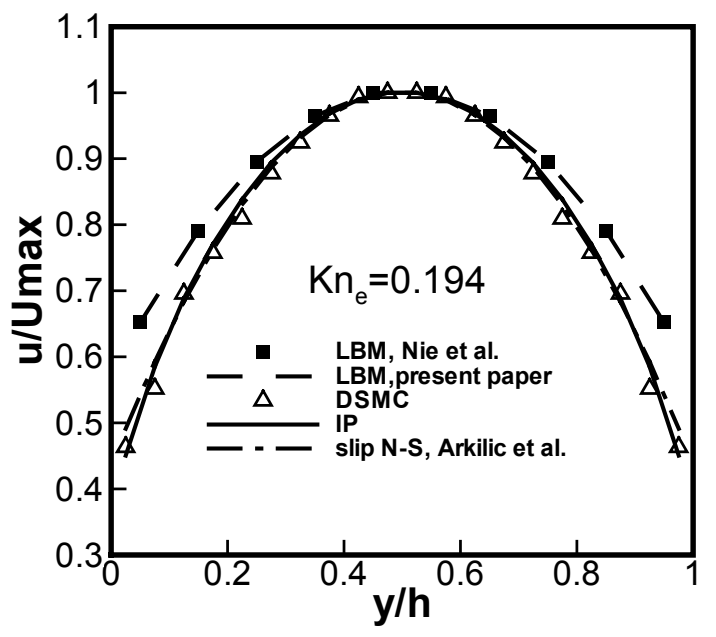

Fig. 4 Comparison of the LBM, DSMC, IP, and slip Navier-Stokes velocity profiles at the channel exit for $K n=0.194$ and. $\mathrm{p}_{\mathrm{i}} / \mathrm{p}_{\mathrm{e}}=2.0$

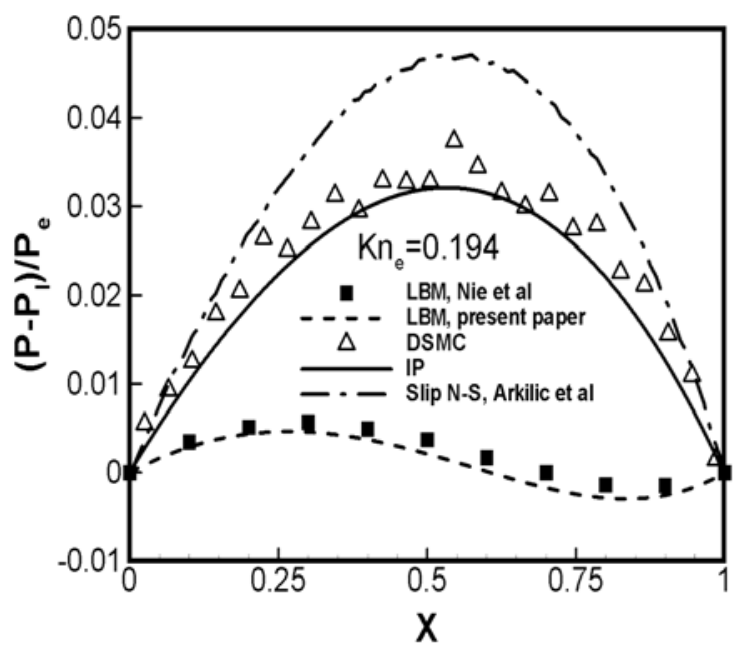

Fig. 5 Comparison of the deviation of the stream-wise pressure from a linear pressure distribution given by LBM, DSMC, IP, and slip Navier-Stokes equation. 


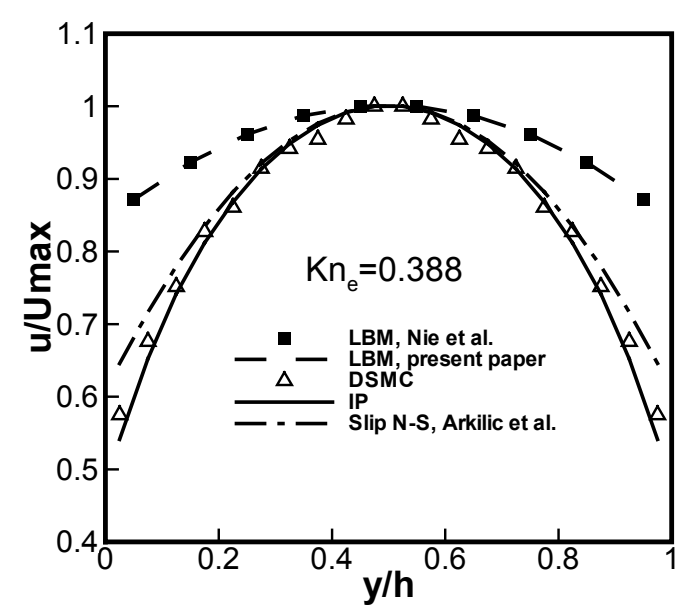

Fig.6 Comparison of the LBM, DSMC, IP, and slip Navier-Stokes velocity profiles at the channel exit for $K n=0.388, \mathrm{p}_{\mathrm{i}} / \mathrm{p}_{\mathrm{e}}=2.0$

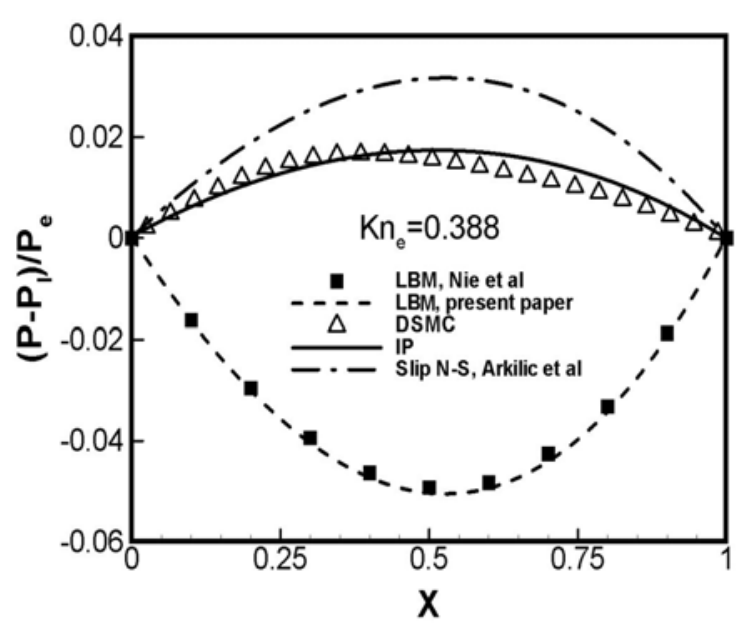

Fig.7 Deviation of pressure distribution from linearly Comparison of LBM, DSMC and IP simulations, $K n=0.388, \mathrm{p}_{\mathrm{i}} / \mathrm{p}_{\mathrm{e}}=2.0$

Table 1

Sample sizes used to yield the DSMC and IP data

\begin{tabular}{cccc}
\hline $\begin{array}{c}\text { Pressure ratio } \\
\mathrm{p}_{\mathrm{i}} / \mathrm{p}_{\mathrm{e}}\end{array}$ & $\begin{array}{c}\text { Knudsen number } \\
K n\end{array}$ & $\begin{array}{c}\text { Sample size for } \\
\text { DSMC }\end{array}$ & $\begin{array}{c}\text { Sample size for } \\
\text { IP }\end{array}$ \\
\hline 1.4 & 0.0194 & $6.6 \times 10^{6}$ & $7.4 \times 10^{4}$ \\
2 & 0.0194 & $1 \times 10^{7}$ & $7.5 \times 10^{4}$ \\
2 & 0.194 & $6 \times 10^{6}$ & $1.4 \times 10^{4}$ \\
2 & 0.388 & $5.6 \times 10^{6}$ & $1.4 \times 10^{4}$ \\
\hline
\end{tabular}

and predict wrong trend in the pressure deviation from linearity. Thus the present paper by using the well-tested DSMC method has shown the unfeasibility of using the Lattice Boltzmann method to simulate transitional flow in MEMS. It is desirable to have experimental data to compare with the results of DSMC, LBM and IP. But the available experimental investigations have been performed exclusively for long channels with a length to height ratio of 2500-5600 (Pong, et al., 1994; Shih, et al., 1996; Arkilic, et al., 1997) where IP has shown good agreement but DSMC calculation has not been performed for the difficulty with unattainable computation time. The desired overall comparison will be hopefully available in the future.

\section{ACKNOWLEDGMENT}

The authors appreciate support from NNSFC under Grants 19889209 and 90205024.

\section{NOMENCLATURE}

$\mathrm{a}$

constant in definition of mean free path

$\begin{array}{cl}\mathrm{c}_{\mathrm{s}} & \text { sound speed, } \mathrm{m} / \mathrm{s} \\ \mathbf{c}_{\mathrm{i}} & \text { particle velocities in LBM, } \mathrm{m} / \mathrm{s} \\ \mathrm{f} & \text { distribution function } \\ \mathrm{f}_{\mathrm{i}} & \text { distribution function with velocity } \mathrm{c}_{\mathrm{i}} \\ \mathrm{h} & \text { the height of the micro channel, } \mathrm{m} \\ K n & \text { Knudsen number, } \lambda / \mathrm{h} \\ \mathrm{l} & \text { the length of the micro channel, } \mathrm{m} \\ \dot{\mathrm{M}} & \text { flowrate across the channel, } \mathrm{kg} / \mathrm{s} \\ \mathrm{p} & \text { pressure, } \mathrm{N} / \mathrm{m}^{2} \\ \mathrm{p}_{\mathrm{e}} & \text { pressure at the exit of the channel, } \\ & \mathrm{N} / \mathrm{m}^{2} \\ \mathrm{p}_{\mathrm{i}} & \text { pressure at the inlet of the channel, } \\ & \mathrm{N} / \mathrm{m}^{2} \\ \mathrm{p}_{1} & \text { linearly distribution } \\ \mathrm{r} & \begin{array}{l}\mathrm{p}_{1}=\mathrm{p}_{\mathrm{e}}+\left(\mathrm{p}_{\mathrm{i}}-\mathrm{p}_{\mathrm{e}}\right)(1-\mathrm{X}) \\ \mathrm{t}\end{array} \quad \begin{array}{l}\text { particle spatial location } \\ \mathrm{u}\end{array} \quad \text { velocity along the axis of the channel, }\end{array}$




\begin{tabular}{|c|c|}
\hline & $\mathrm{m} / \mathrm{s}$ \\
\hline $\mathbf{u}$ & velocity, $\mathrm{m} / \mathrm{s}$ \\
\hline $\mathrm{U}_{\max }$ & $\begin{array}{l}\text { maximum velocity of the cross } \\
\text { section, } \mathrm{m} / \mathrm{s}\end{array}$ \\
\hline $\mathrm{x}$ & $\begin{array}{l}\text { the Cartesian coordinate along the } \\
\text { channel axis, } m\end{array}$ \\
\hline $\mathrm{X}$ & $\begin{array}{l}\text { dimensionless coordinate along the } \\
\text { channel axis, } X=x / 1\end{array}$ \\
\hline$\delta \mathrm{t}$ & time step, $\mathrm{s}$ \\
\hline$\lambda$ & mean free path of the molecules, $\mathrm{m}$ \\
\hline$\rho$ & density, $\mathrm{kg} / \mathrm{m}^{3}$ \\
\hline$\tau$ & $\begin{array}{l}\text { relaxation factor in the BGK model } \\
\text { equation }\end{array}$ \\
\hline$\tau^{\prime}$ & modified relaxation factor \\
\hline
\end{tabular}

Subscripts

$\alpha, \beta \quad$ Indicate the spatial directions in Cartesian coordinates

i Indicate the directions of the moving velocities along the links between the lattice notes

\section{REFERENCES}

Arkilic, E.B., Schmidt, M.A, and Breuer, K.S., 1997, TMAC Measurement in Silicon Micromachined Channels, Rarefied Gas Dynamics, ed. C.Shen, Peking University Press, pp. 983-988.

Bhatnangar, P.L., Gross, E.P., and Krook, M., 1954, Model for Collision Processes in Gases. I. Small Amplitude Processes in Charged and Neutral One-Component Systems, Physical Review, Vol. 94, pp.511-525.

Bird, G..A., 1994, Molecular Gas Dynamics and Direct Simulation of Gas Flows, Clarendon Press, Oxford.

Cai, C., Boyd, I. D., Fan, J., and Candler G. V., 2000, Direct Simulation Methods for Low-Speed Microchannel Flows, J. Thermophysics and Heat Transfer, Vol. 14, pp. 368-378.

Cercignani, C., 1998, The Boltzmann Equation and its Applications, Springer, New York.

Chen, S., Martinez, D., and Mei, R. W. 1996, On Boundary Condition in Lattice Boltzmann Methods, Phys. Fluids, Vol.8, pp. 2527-2536.

Chen, S., and Doolen, G.D., 1998, Lattice Boltzmann Method for Fluid Flows, Ann. Rev. Fluid Mechanics, Vol.30, pp. 329-364.
Fan, J., Boyd, I. D., and Cai, C. P., 2001, Computation of Rarefied Flows around a NACA 0012 Airfoil, AIAA J., Vol. 39, pp. 618-625.

Fan, J., and Shen, C., 1999, Statistical Simulation of Low-speed Unidirectional Flow in Transition Regime, Rarefied Gas Dynamics, eds. by R. Brun et al., Vol.2, pp.245-252.

Fan, J., and Shen, C., 2001, Statistical Simulation of Low-speed Rarefied Gas Flows, Journal of.Computational Physics, Vol.167, pp.393-412.

Ho, C.M., and Tai, Y.C., 1998, Micro-electro-mechanical System (MEMS) and Fluid Flows, Ann Rev. Fluid Mech., Vol.30, pp.579-612.

Jiang, J., Shen, C., and Fan, J., 2003, Statistical Simulation of Non-circular Cross Section Poiseuille Flows, presented at this conference.

Karniadakis, G.E., and Beskok, A., 2001, Micro Flows, Fundamentals and Simulation, Springer, New York.

Nie, X., Doolen, G.D., and Chen, S., 2002, Lattice Boltzmann Simulation of Fluid Flow in MEMS, Journal of Statistical Physics, Vol.107, pp.279-289.

Ohwada,T., Sone, Y., and Aoki, K., 1989, Numerical Analysis of The Poiseuille and Thermal transitional Flows Between Two Parallel Plates on The Basis of The Boltzmann Equation for Hard Sphere Molecules, Physics of Fluid A, Vol.1, pp.2042-2049.

Pong, K. C., Ho, C. M., Liu, J.Q., and Tai, Y. C., 1994, Non-linear Pressure Distribution in Uniform Microchannel, ASME-FED, Vol.197, pp51-56.

Qian, Y. H., d'Humiéres, D., and Lallemand, P., 1992, Lattice BGK Models for Navier-Stokes Equation, EuroPhysics Letters, Vol.17, pp.479-484.

Sharipov, F., and Seleznev, V., 1998, Journal of .Physical and Chemical Reference Data, Vol.27, pp. $657-706$.

Shen,C.,2003, Rarefied Gas Dynamics, National Defense Industry Press, Beijing (in Chinese).

Shen, C., Fan, J. and Xie, C., 2003, Statistical Simulation of Rarefied Gas Flows in Micro channels, to appear in Journal of.Computational Physics.

Shih, J.C., Ho, C. M., Liu, J. Q., and Tai, Y. C., 1996, Monoatomic and Polyatomic Gas Flow through Uniform Microchannels, ASME-DSC, Vol.59, pp.197-203.

Sun, Q.H., and Boyd, I.D., 2002, A Direct Simulation Method for Subsonic, Microscale Gas Flows, J. Comp. Phys., Vol. 179, pp. 400-425.

Sun, Q., Boyd, I. D., and Candler, G. V., 2001, Numerical Simulation of Gas Flow over Micro-Scale Airfoils, AIAA 2001-3071.

Xie, C., Fan, J., and Shen, C., 2002, Rarefied Gas Flows in Micro Channels, presented at 23rd International Symposium on Rarefied Gas Dynamics, 21-25 July, 2002, Whistler. 